\title{
ON MIXED-TYPE REVERSE-ORDER LAWS FOR THE MOORE-PENROSE INVERSE OF A MATRIX PRODUCT
}

\author{
YONGGE TIAN
}

Received 13 January 2003

\begin{abstract}
Some mixed-type reverse-order laws for the Moore-Penrose inverse of a matrix product are established. Necessary and sufficient conditions for these laws to hold are found by the matrix rank method. Some applications and extensions of these reverse-order laws to the weighted Moore-Penrose inverse are also given.
\end{abstract}

2000 Mathematics Subject Classification: 15A03, 15A09.

If $A$ and $B$ are a pair of invertible matrices of the same size, then the product $A B$ is nonsingular, too, and the inverse of the product $A B$ satisfies the reverse-order law $(A B)^{-1}=B^{-1} A^{-1}$. This law can be used to find the properties of $(A B)^{-1}$, as well as to simplify various matrix expressions that involve the inverse of a matrix product. However, this formula cannot trivially be extended to the Moore-Penrose inverse of matrix products. For a general $m \times n$ complex matrix $A$, the Moore-Penrose inverse $A^{\dagger}$ of $A$ is the unique $n \times m$ matrix $X$ that satisfies the following four Penrose equations:

(i) $A X A=A$,

(ii) $X A X=X$,

(iii) $(A X)^{*}=A X$,

(iv) $(X A)^{*}=X A$,

where $(\cdot)^{*}$ denotes the conjugate transpose of a complex matrix. A matrix $X$ is called a $\{1\}$-inverse (inner inverse) of $A$ if it satisfies (i) and is denoted by $A^{-}$. General properties of the Moore-Penrose inverse can be found in [2, 4, 16].

Let $A$ and $B$ be a pair of matrices such that $A B$ exists. In many situations, one needs to find the Moore-Penrose inverse of the product $A B$ and its properties. Because $A^{\dagger} A, B B^{\dagger}$, and $B B^{\dagger} A^{\dagger} A$ are not necessarily identity matrices, the relationship between $(A B)^{\dagger}$ and $B^{\dagger} A^{\dagger}$ is quite complicated and the reverse-order law $(A B)^{\dagger}=B^{\dagger} A^{\dagger}$ does not necessarily hold. Therefore, it is not easy to simplify matrix expressions that involve the MoorePenrose inverse of matrix products. Theoretically speaking, for any matrix product $A B$, the Moore-Penrose inverse $(A B)^{\dagger}$ can be written as

$$
(A B)^{\dagger}=B^{\dagger} A^{\dagger} \quad \text { or } \quad(A B)^{\dagger}=B^{\dagger} A^{\dagger}+X,
$$

where $X$ is a residue matrix. For these two situations, one can consider the following two problems:

(I) necessary and sufficient conditions for $(A B)^{\dagger}=B^{\dagger} A^{\dagger}$ to hold, 
(II) if $(A B)^{\dagger} \neq B^{\dagger} A^{\dagger}$, find possible expressions of $X$ in $(A B)^{\dagger}=B^{\dagger} A^{\dagger}+X$, and then determine necessary and sufficient conditions for $(A B)^{\dagger}=B^{\dagger} A^{\dagger}+X$ to hold.

The investigation of the Moore-Penrose inverse of the product $A B$ was started in 1960s. For the standard situation $(A B)^{\dagger}=B^{\dagger} A^{\dagger}$, a well-known result due to Greville [9] asserts that

$$
(A B)^{\dagger}=B^{\dagger} A^{\dagger} \Longleftrightarrow \mathcal{R}\left(A^{*} A B\right) \subseteq \mathcal{R}(B), \quad \mathcal{R}\left(B B^{*} A^{*}\right) \subseteq \mathcal{R}\left(A^{*}\right),
$$

where $\mathcal{R}(\cdot)$ denotes the range (column space) of a matrix. Many other equivalent conditions for $(A B)^{\dagger}=B^{\dagger} A^{\dagger}$ to hold can be found in [2, 4, 16, 26]. Generally speaking, the two range inclusions in (2) are strict conditions for any pair of matrices $A$ and $B$ to satisfy. Therefore, it is necessary to seek various weaker reverse-order laws for $(A B)^{\dagger}$ to satisfy. In addition to $(2),(A B)^{\dagger}$ may satisfy some other mixed-type reverse-order laws. For example,

$$
\begin{gathered}
(A B)^{\dagger}=\left(A^{\dagger} A B\right)^{\dagger}\left(A B B^{\dagger}\right)^{\dagger}, \\
(A B)^{\dagger}=B^{\dagger}\left(A^{\dagger} A B B^{\dagger}\right)^{\dagger} A^{\dagger}, \quad(A B)^{\dagger}=B^{*}\left(A^{*} A B B^{*}\right)^{\dagger} A^{*} .
\end{gathered}
$$

These reverse-order laws were studied in [6, 8, 11, 26]. Although these matrix equalities are more complicated than the law in (2), the conditions for these equalities to hold are weaker than that for (2) to hold. In fact, mixed-type reverse-order laws also stem from various reasonable operations for the Moore-Penrose inverse of matrix products (see Remark 10). Although $(A B)^{\dagger}$ can be written as $(A B)^{\dagger}=B^{\dagger} A^{\dagger}+X$ in general, it is not easy to give an explicit expression for the residue matrix $X$ for the given matrices $A$ and $B$. Some discussion for the expression of $X$ and its properties were given in [8].

In the investigation of $(A B)^{\dagger}$, we observe that a possible expression for $(A B)^{\dagger}$ is

$$
(A B)^{\dagger}=B^{\dagger} A^{\dagger}-B^{\dagger}\left[\left(I_{n}-B B^{\dagger}\right)\left(I_{n}-A^{\dagger} A\right)\right]^{\dagger} A^{\dagger} .
$$

A direct motivation for us to find out the residue matrix in (4) arises from two different decompositions of the following block matrix:

$$
M=\left[\begin{array}{ll}
I_{n} & B \\
A & 0
\end{array}\right]
$$

and its generalized inverses. In fact, it is easy to verify that $M$ can be decomposed as the following two forms:

$$
\begin{gathered}
M=\left[\begin{array}{cc}
I_{n} & 0 \\
A & I_{m}
\end{array}\right]\left[\begin{array}{cc}
I_{n} & 0 \\
0 & -A B
\end{array}\right]\left[\begin{array}{cc}
I_{n} & B \\
0 & I_{k}
\end{array}\right]:=P_{1} N_{1} Q_{1}, \\
M= \\
{\left[\begin{array}{cc}
I_{n} & \left(I_{n}-B B^{\dagger}\right) A^{\dagger} \\
0 & I_{m}
\end{array}\right]\left[\begin{array}{cc}
T & B \\
A & 0
\end{array}\right]\left[\begin{array}{cc}
I_{n} & 0 \\
B^{\dagger} & I_{k}
\end{array}\right]:=P_{2} N_{2} Q_{2},}
\end{gathered}
$$


where $T=\left(I_{n}-B B^{\dagger}\right)\left(I_{n}-A^{\dagger} A\right)$. From these two decompositions, one can find two $\{1\}$-inverses of $M$ as follows:

$$
\begin{aligned}
M^{-} & =Q_{1}^{-1} N_{1}^{\dagger} P_{1}^{-1}=\left[\begin{array}{cc}
I_{n} & -B \\
0 & I_{k}
\end{array}\right]\left[\begin{array}{cc}
I_{n} & 0 \\
0 & -(A B)^{\dagger}
\end{array}\right]\left[\begin{array}{cc}
I_{n} & 0 \\
-A & I_{m}
\end{array}\right] \\
& =\left[\begin{array}{cc}
I_{n} & B(A B)^{\dagger} \\
(A B)^{\dagger} A & -(A B)^{\dagger}
\end{array}\right], \\
M^{-} & =Q_{2}^{-1} N_{2}^{\dagger} P_{2}^{-1}=\left[\begin{array}{cc}
I_{n} & 0 \\
-B^{\dagger} & I_{k}
\end{array}\right]\left[\begin{array}{cc}
T^{\dagger} & A^{\dagger} \\
B^{\dagger} & 0
\end{array}\right]\left[\begin{array}{cc}
I_{n} & -\left(I_{n}-B B^{\dagger}\right) A^{\dagger} \\
0 & I_{m}
\end{array}\right] \\
& =\left[\begin{array}{cc}
T^{\dagger} & A^{\dagger}-T^{\dagger} A^{\dagger} \\
B^{\dagger}-B^{\dagger} T^{\dagger} & B^{\dagger} T^{\dagger} A^{\dagger}-B^{\dagger} A^{\dagger}
\end{array}\right] .
\end{aligned}
$$

These two $\{1\}$-inverses of $M$ are not necessarily equal. Therefore, it is natural to consider under what conditions the two $\{1\}$-inverses of $M$ in (7) are equal; or some blocks of them are equal. The mixed-type reverse-order law (4) is noticed by comparing the lower right blocks of (7).

Because the right-hand side of (4) involves complicated matrix operations, it is not easy to establish necessary and sufficient conditions for (4) to hold by definitions, as well as various matrix decompositions associated with $A, B$, and $A B$. In the investigation of various problems on generalized inverses of matrices, the present author notices that the rank of matrix is a simple and powerful method for dealing with the relationship between any two matrix expressions involving generalized inverses. In fact, any two matrices $A$ and $B$ of the same size are equal if and only if $r(A-B)=0$, where $r(\cdot)$ denotes the rank of a matrix. If one can find some nontrivial formulas for the rank of $A-B$, then necessary and sufficient conditions for $A=B$ to hold can be derived from these rank formulas. This method can be used for investigating the relations between any two matrix expressions that involve generalized inverses. Several simple rank formulas for the differences of matrices found by the present author are given below:

$$
\begin{gathered}
r\left(A^{k} A^{\dagger}-A^{\dagger} A^{k}\right)=r\left[\begin{array}{l}
A^{k} \\
A^{*}
\end{array}\right]+r\left[A^{k}, A^{*}\right]-2 r(A), \\
r\left(A^{*} A^{\dagger}-A^{\dagger} A^{*}\right)=r\left(A A^{*} A^{2}-A^{2} A^{*} A\right), \\
r\left(A B-A B B^{\dagger} A^{\dagger} A B\right)=r\left[A^{*}, B\right]+r(A B)-r(A)-r(B), \\
r\left([A, B]^{\dagger}-\left[\begin{array}{c}
A^{\dagger} \\
B^{\dagger}
\end{array}\right]\right)=r\left[A A^{*} B, B B^{*} A\right], \\
r\left([A, B]^{\dagger}[A, B]-\left[\begin{array}{cc}
A^{\dagger} A & 0 \\
0 & B^{\dagger} B
\end{array}\right]\right)=r(A)+r(B)-r[A, B], \\
\min _{A^{-}, B^{-}} r\left(A^{-}-B^{-}\right)=r(A-B)-r\left[\begin{array}{c}
A \\
B
\end{array}\right]-r[A, B]+r(A)+r(B),
\end{gathered}
$$


where $[A, B]$ denotes a row block matrix; see Tian $[20,23,25,26]$. The significance of these simple rank formulas is: they connect different matrix expressions through the rank of these matrices. From these rank equalities, one can derive some basic properties for the matrices on the left-hand sides. For instance, let the right-hand sides of the above rank equalities be zero and simplify by some elementary methods, one can immediately obtain necessary and sufficient conditions for the matrices on the left-hand sides to be zero.

In this paper, we establish a rank formula associated with (4) and then derive from the rank formula a necessary and sufficient condition for (4) to hold.

The following rank formulas are well known:

$$
\begin{aligned}
r[A, B] & =r(A)+r\left(B-A A^{\dagger} B\right)=r(B)+r\left(A-B B^{\dagger} A\right), \\
r\left[\begin{array}{ll}
A & B \\
C & 0
\end{array}\right] & =r(B)+r(C)+r\left[\left(I-B B^{\dagger}\right) A\left(I-C^{\dagger} C\right)\right] ;
\end{aligned}
$$

if $\mathcal{R}(B) \subseteq \mathcal{R}(A)$ and $\mathcal{R}\left(C^{*}\right) \subseteq \mathcal{R}\left(A^{*}\right)$, then

$$
r\left[\begin{array}{ll}
A & B \\
C & D
\end{array}\right]=r(A)+r\left(D-C A^{\dagger} B\right),
$$

see Marsaglia and Styan [12].

Recall the equality $A^{*}\left(A^{*} A A^{*}\right)^{\dagger} A^{*}=A^{\dagger}$ (see Zlobec [28]) and notice that $\mathcal{R}(A)=$ $\mathcal{R}\left(A A^{*} A\right)$ and $\mathcal{R}\left(A^{*}\right)=\mathcal{R}\left(A^{*} A A^{*}\right)$. By appealing to (11), the rank of the Schur complement $D-C A^{\dagger} B$ is

$$
r\left(D-C A^{\dagger} B\right)=r\left[\begin{array}{cc}
A^{*} A A^{*} & A^{*} B \\
C A^{*} & D
\end{array}\right]-r(A) .
$$

This rank equality is quite useful in dealing with various matrix expressions involving the Moore-Penrose inverse. Rank formulas for the Schur complement $D-C A^{-} B$, where $A^{-}$is a $\{1\}$-inverse of $A$, can be found in [23].

The main result of this paper is given below.

TheOREM 1. Let $A \in \mathbb{C}^{m \times n}$ and $B \in \mathbb{C}^{n \times p}$, and let $T=\left(I_{n}-B B^{\dagger}\right)\left(I_{n}-A^{\dagger} A\right)$. Then,

$$
r\left[(A B)^{\dagger}-B^{\dagger} A^{\dagger}+B^{\dagger} T^{\dagger} A^{\dagger}\right]=r\left[\begin{array}{c}
A B \\
A B B^{*} B
\end{array}\right]+r\left[A B, A A^{*} A B\right]-2 r(A B) .
$$

Hence, the reverse-order law (4) holds if and only if A and B satisfy the following two range equalities:

$$
\mathcal{R}\left(A A^{*} A B\right)=\mathcal{R}(A B), \quad \mathcal{R}\left[\left(A B B^{*} B\right)^{*}\right]=\mathcal{R}\left[(A B)^{*}\right] .
$$

Proof. From (12), we first obtain

$$
r\left[(A B)^{\dagger}-B^{\dagger} A^{\dagger}+B^{\dagger} T^{\dagger} A^{\dagger}\right]=r\left[\begin{array}{cc}
T^{*} T T^{*} & T^{*} A^{\dagger} \\
B^{\dagger} T^{*} & B^{\dagger} A^{\dagger}-(A B)^{\dagger}
\end{array}\right]-r(T) .
$$


Applying (10) to $T$ yields

$$
r(T)=r\left[\begin{array}{cc}
I_{n} & B \\
A & 0
\end{array}\right]-r(A)-r(B)=n+r(A B)-r(A)-r(B)
$$

It is easy to verify that $T^{*} T T^{*}=\left(I_{n}-A^{\dagger} A\right) T\left(I_{n}-B B^{\dagger}\right)$. Recall that elementary matrix operations and block elementary matrix operations do not change the rank of matrix. Thus, we can find by (10) and block elementary matrix operations that

$$
\begin{aligned}
& r\left[\begin{array}{cc}
T^{*} T T^{*} & T^{*} A^{\dagger} \\
B^{\dagger} T^{*} & B^{\dagger} A^{\dagger}-(A B)^{\dagger}
\end{array}\right] \\
& =r\left[\begin{array}{cc}
\left(I_{n}-A^{\dagger} A\right) T\left(I_{n}-B B^{\dagger}\right) & \left(I_{n}-A^{\dagger} A\right)\left(I_{n}-B B^{\dagger}\right) A^{\dagger} \\
B^{\dagger}\left(I_{n}-A^{\dagger} A\right)\left(I_{n}-B B^{\dagger}\right) & B^{\dagger} A^{\dagger}-(A B)^{\dagger}
\end{array}\right] \\
& =r\left[\begin{array}{ccc}
T & \left(I_{n}-B B^{\dagger}\right) A^{\dagger} & A^{\dagger} \\
B^{\dagger}\left(I_{n}-A^{\dagger} A\right) & B^{\dagger} A^{\dagger}-(A B)^{\dagger} & 0 \\
B^{\dagger} & 0 & 0
\end{array}\right]-r(A)-r(B) \\
& =r\left[\begin{array}{ccc}
I_{n}+B B^{\dagger} A^{\dagger} A & -B B^{\dagger} A^{\dagger} & A^{\dagger} \\
-B^{\dagger} A^{\dagger} A & B^{\dagger} A^{\dagger}-(A B)^{\dagger} & 0 \\
B^{\dagger} & 0 & 0
\end{array}\right]-r(A)-r(B) \\
& =r\left[\begin{array}{ccc}
I_{n} & -B B^{\dagger} A^{\dagger} & A^{\dagger} \\
-(A B)^{\dagger} A & B^{\dagger} A^{\dagger}-(A B)^{\dagger} & 0 \\
B^{\dagger} & 0 & 0
\end{array}\right]-r(A)-r(B) \\
& =r\left[\begin{array}{ccc}
I_{n} & 0 & 0 \\
0 & B^{\dagger} A^{\dagger}-(A B)^{\dagger}-(A B)^{\dagger} A B B^{\dagger} A^{\dagger} & (A B)^{\dagger} \\
0 & B^{\dagger} A^{\dagger} & -B^{\dagger} A^{\dagger}
\end{array}\right]-r(A)-r(B) \\
& =n+r\left[\begin{array}{cc}
B^{\dagger} A^{\dagger}-(A B)^{\dagger} A B B^{\dagger} A^{\dagger} & (A B)^{\dagger} \\
0 & -B^{\dagger} A^{\dagger}
\end{array}\right]-r(A)-r(B) .
\end{aligned}
$$

Also note that $\mathcal{R}\left\{\left[I_{n}-(A B)^{\dagger} A B\right] B^{\dagger} A^{\dagger}\right\} \cap \mathcal{R}\left[(A B)^{\dagger}\right]=\{0\}$. It follows that

$$
\mathcal{R}\left[\begin{array}{c}
B^{\dagger} A^{\dagger}-(A B)^{\dagger} A B B^{\dagger} A^{\dagger} \\
0
\end{array}\right] \cap \mathcal{R}\left[\begin{array}{c}
(A B)^{\dagger} \\
-B^{\dagger} A^{\dagger}
\end{array}\right]=\{0\} .
$$

Thus,

$$
\begin{aligned}
r\left[\begin{array}{cc}
B^{\dagger} A^{\dagger}-(A B)^{\dagger} A B B^{\dagger} A^{\dagger} & (A B)^{\dagger} \\
0 & -B^{\dagger} A^{\dagger}
\end{array}\right] \\
\quad=r\left[\begin{array}{c}
B^{\dagger} A^{\dagger}-(A B)^{\dagger} A B B^{\dagger} A^{\dagger} \\
0
\end{array}\right]+r\left[\begin{array}{c}
(A B)^{\dagger} \\
-B^{\dagger} A^{\dagger}
\end{array}\right] \\
=r\left[B^{\dagger} A^{\dagger}-(A B)^{\dagger} A B B^{\dagger} A^{\dagger}\right]+r\left[\begin{array}{c}
(A B)^{\dagger} \\
B^{\dagger} A^{\dagger}
\end{array}\right] .
\end{aligned}
$$


Applying (9) gives

$$
\begin{aligned}
r\left[B^{\dagger} A^{\dagger}-(A B)^{\dagger}(A B) B^{\dagger} A^{\dagger}\right] & =r\left[(A B)^{\dagger}, B^{\dagger} A^{\dagger}\right]-r\left[(A B)^{\dagger}\right] \\
& =r\left[(A B)^{*}, B^{\dagger} A^{*}\right]-r(A B) \\
& =r\left[B B^{*}(A B)^{*}, B^{*} A^{*}\right]-r(A B) \\
& =r\left[\begin{array}{c}
A B B^{*} B \\
A B
\end{array}\right]-r(A B), \\
r\left[\begin{array}{c}
(A B)^{\dagger} \\
B^{\dagger} A^{\dagger}
\end{array}\right]=r\left[\begin{array}{c}
(A B)^{*} \\
B^{*} A^{\dagger}
\end{array}\right] & =r\left[\begin{array}{c}
(A B)^{*} A A^{*} \\
B^{*} A^{*}
\end{array}\right]=r\left[A A^{*} A B, A B\right] .
\end{aligned}
$$

Substituting (20) into (19), and (19) into (17), and then (16) and (17) into (15) gives us (13). Let the right-hand side of (13) be zero and note that

$$
r(A B)=r\left(A A^{*} A B\right)=r\left(A B B^{*} B\right) .
$$

Then the equivalence of (4) and (14) follows.

The establishment of (13) is not easy, because the matrix expression on the left-hand side of (13) involves three terms consisting of the Moore-Penrose inverse and the righthand side of (13) involve ranks of block matrices with the products $A B, A A^{*} A B$, and $A B B^{*} B$. However, the two block matrices on the right-hand side of (13) and the two range equalities in (14) are easy to simplify when $A$ and $B$ satisfy some conditions. For example, if both $A$ and $B$ are partial isometries, that is, $A^{\dagger}=A^{*}$ and $B^{\dagger}=B^{*}$, then (14) is satisfied. In this case, (4) becomes

$$
(A B)^{\dagger}=(A B)^{*}-B^{*}\left[\left(I_{n}-B B^{*}\right)\left(I_{n}-A^{*} A\right)\right]^{\dagger} A^{*} .
$$

The most valuable consequence of (4) is concerned with the Moore-Penrose inverse of the product of two orthogonal projectors.

COROLLARY 2. Let $P$ and $Q$ be a pair of orthogonal projectors of order $n$. Then, the product $P Q$ satisfies the following two identities:

$$
\begin{aligned}
& (P Q)^{\dagger}=Q P-Q\left(Q^{\perp} P^{\perp}\right)^{\dagger} P, \\
& (P Q)^{2}=P Q+P Q\left(Q^{\perp} P^{\perp}\right)^{\dagger} P Q
\end{aligned}
$$

where $P^{\perp}=I_{n}-P$ and $Q^{\perp}=I_{n}-Q$. In particular,

$$
\begin{aligned}
& (P Q)^{\dagger}=Q P \Longleftrightarrow Q\left(Q^{\perp} P^{\perp}\right)^{\dagger} P=0, \\
& (P Q)^{2}=P Q \Longleftrightarrow P Q\left(Q^{\perp} P^{\perp}\right)^{\dagger} P Q=0 .
\end{aligned}
$$

Proof. Note that $P^{2}=P=P^{*}=P^{\dagger}, Q^{2}=Q=Q^{*}=Q^{\dagger}$ for any pair of orthogonal projectors $P$ and $Q$. Thus, $P$ and $Q$ satisfy (14), and (4) is reduced to (23). Premultiplying and postmultiplying both sides of (23) by $P Q$ yield (24). 
Recall that for any pair of orthogonal projectors $P$ and $Q$,

$$
(P Q)^{\dagger}=Q P \Longleftrightarrow(P Q)^{2}=P Q \Longleftrightarrow P Q=Q P,
$$

see, for example, [1]. Thus, (25) could be regarded as two new equivalent statements for the commutativity of two orthogonal projectors. There are many results in the literature on products of orthogonal projectors and related topics; see, for example, [1, 13]. The two identities (23) and (24) are two fundamental results for the product of two orthogonal projectors. They can be used for dealing with various matrix expressions that involve products of two orthogonal projectors. For example, the product $P Q P$ satisfies the following identity:

$$
\begin{aligned}
(P Q P)^{\dagger} & =\left[(P Q)(P Q)^{*}\right]^{\dagger}=(Q P)^{\dagger}(P Q)^{\dagger} \\
& =P\left[I_{n}-\left(P^{\perp} Q^{\perp}\right)^{\dagger}\right] Q\left[I_{n}-\left(Q^{\perp} P^{\perp}\right)^{\dagger}\right] P .
\end{aligned}
$$

Moreover, it is easy to verify that

$$
\left[(P Q)^{2}\right]^{\dagger}=(P Q)^{\dagger}(Q P)^{\dagger}(P Q)^{\dagger}, \quad(P Q)^{\#}=(Q P)^{\dagger}(P Q)^{\dagger}(Q P)^{\dagger},
$$

where $(P Q)^{\#}$ is the group inverse of $P Q$. Hence, one can also derive from (23) two identities for $\left[(P Q)^{2}\right]^{\dagger}$ and $(P Q)^{\#}$. From (23), one can also derive some valuable expressions for $(P \pm Q)^{\dagger}$ and $(P Q \pm Q P)^{\dagger}$; see [5].

Let $\|A\|$ denote the spectral norm of a matrix $A$, that is, the maximal singular value of $A$. For a nonnull orthogonal projector $P,\|P\|=1$. For any pair of orthogonal projectors $P$ and $Q$ with $P Q \neq 0$, it can be derived from (23) the following norm equality:

$$
\left\|(P Q)^{\dagger}\right\| \leq\left\|I_{n}-\left(Q^{\perp} P^{\perp}\right)^{\dagger}\right\| .
$$

It was shown in $[3,15]$ that if $P$ is idempotent with $P \neq 0$ and $P \neq I$, then $\|I-P\|=\|P\|$. If $P$ and $Q$ are two orthogonal projectors, then $(P Q)^{\dagger}$ is idempotent; see [14]. Note that $I_{n}-P$ and $I_{n}-Q$ are orthogonal projectors. Hence, $\left[\left(I_{n}-Q\right)\left(I_{n}-P\right)\right]^{\dagger}$ is idempotent. Thus, if $\left(I_{n}-Q\right)\left(I_{n}-P\right) \neq 0$, then

$$
\left\|I_{n}-\left(Q^{\perp} P^{\perp}\right)^{\dagger}\right\|=\left\|\left(Q^{\perp} P^{\perp}\right)^{\dagger}\right\| .
$$

Applying this equality to (29), we see that if $P Q \neq 0$ and $Q^{\perp} P^{\perp} \neq 0$, then

$$
\left\|(P Q)^{\dagger}\right\| \leq\left\|\left(Q^{\perp} P^{\perp}\right)^{\dagger}\right\| .
$$

Replacing $P$ with $I_{n}-Q$ and $Q$ with $I_{n}-P$ in (31) also gives

$$
\left\|\left(Q^{\perp} P^{\perp}\right)^{\dagger}\right\| \leq\left\|(P Q)^{\dagger}\right\| .
$$

Hence, we have the following result.

THEOREM 3. Let $P$ and $Q$ be a pair of orthogonal projectors with both $P Q \neq 0$ and $Q^{\perp} P^{\perp} \neq 0$. Then,

$$
\left\|(P Q)^{\dagger}\right\|=\left\|\left(Q^{\perp} P^{\perp}\right)^{\dagger}\right\|
$$


Identity (23) can be used to establish some identities for the Moore-Penrose inverse of $A B C$, where $A, B$, and $C$ are three orthogonal projectors.

THEOREM 4. Let $A, B$, and $C$ be a triple of orthogonal projectors of order $n$. Then,

$$
\begin{gathered}
(A B C)^{\dagger}=\left(P_{(A B) * C}\right)^{\dagger}\left(A P_{B C}\right)^{\dagger}, \\
(A B C)^{\dagger}=C\left[I_{n}-\left(C^{\perp} P_{(A B) *}^{\perp}\right)^{\dagger}\right](A B)^{\dagger} B(B C)^{\dagger}\left[I_{n}-\left(P_{B C}^{\perp} A^{\perp}\right)^{\dagger}\right] A,
\end{gathered}
$$

where $P_{(A B)} *=(A B)^{\dagger} A B$ and $P_{B C}=B C(B C)^{\dagger}$.

Proof. Recall a simple result in [7] that any matrix product $U V$ satisfies

$$
(U V)^{\dagger}=\left(U^{\dagger} U V\right)^{\dagger}\left(U V V^{\dagger}\right)^{\dagger} .
$$

Applying this formula to $A B C=(A B)(B C)$ gives (34). Applying (23) to $\left(P_{(A B)} * C\right)^{\dagger}$ and $\left(A P_{B C}\right)^{\dagger}$ also gives

$$
\begin{aligned}
\left(P_{(A B) *} C\right)^{\dagger} & =C P_{(A B)^{*}}-C\left(C^{\perp} P_{(A B)^{*}}^{\perp}\right)^{\dagger} P_{(A B)^{*},} \\
\left(A P_{B C}\right)^{\dagger} & =P_{B C} A-P_{B C}\left(P_{B C}^{\perp} A^{\perp}\right)^{\dagger} A .
\end{aligned}
$$

Substituting these two results into (34) yields (35).

Although reverse-order laws for the Moore-Penrose inverse of matrix products have many different expressions, some of these reverse-order laws may be equivalent. A simple example is

$$
(A B)^{\dagger}=B^{\dagger} A^{\dagger} \Longleftrightarrow\left[\left(A^{\dagger}\right)^{*} B\right]^{\dagger}=B^{\dagger} A^{*} \Longleftrightarrow\left[A\left(B^{\dagger}\right)^{*}\right]^{\dagger}=B^{*} A^{\dagger}
$$

see [21]. When investigating (4), we also find that (4) is equivalent to the following two mixed-type reverse-order laws:

$$
\begin{aligned}
& (A B)^{\dagger}=B^{\dagger}\left(A^{\dagger} A B B^{\dagger}\right)^{\dagger} A^{\dagger}, \\
& (A B)^{\dagger}=B^{*}\left(A^{*} A B B^{*}\right)^{\dagger} A^{*} .
\end{aligned}
$$

The reverse-order law (39) was first studied by Galperin and Waksman [8], and then by Izumino [11] for a product of two linear operators. They showed that (39) holds if and only if

$$
\mathcal{R}\left[\left(A^{*}\right)^{\dagger} B\right]=\mathcal{R}(A B), \quad \mathcal{R}\left(B^{\dagger} A^{*}\right)=\mathcal{R}\left[(A B)^{*}\right] .
$$

This result is also true for a complex matrix product. Because the Moore-Penrose inverses of $A$ and $B$ are contained in the condition (41), it cannot be regarded as a satisfactory necessary and sufficient condition for (39) to hold. In fact, (41) is equivalent to (14) by noticing

$$
\begin{gathered}
r\left[A B,\left(A^{*}\right)^{\dagger} B\right]=r\left[A A^{*} A B, A A^{*}\left(A^{*}\right)^{\dagger} B\right]=r\left[A A^{*} A B, A B\right], \\
r\left[\begin{array}{c}
A B \\
A\left(B^{*}\right)^{\dagger}
\end{array}\right]=r\left[\begin{array}{c}
A B B^{*} B \\
A\left(B^{*}\right)^{\dagger} B^{*} B
\end{array}\right]=r\left[\begin{array}{c}
A B B^{*} B \\
A B
\end{array}\right] .
\end{gathered}
$$


Without much effort, one can show that

$$
r\left[(A B)^{\dagger}-B^{\dagger}\left(A^{\dagger} A B B^{\dagger}\right)^{\dagger} A^{\dagger}\right]=r\left[\begin{array}{c}
A B \\
A B B^{*} B
\end{array}\right]+r\left[A B, A A^{*} A B\right]-2 r(A B) ;
$$

see [26]. Equality (43) is derived from the following result.

LEMMA 5 [22]. Let $X_{1}$ and $X_{2}$ be a pair of outer inverses of a matrix $A$, that is, $X_{1} A X_{1}=$ $X_{1}$ and $X_{2} A X_{2}=X_{2}$. Then,

$$
r\left(X_{1}-X_{2}\right)=r\left[\begin{array}{l}
X_{1} \\
X_{2}
\end{array}\right]+r\left[X_{1}, X_{2}\right]-r\left(X_{1}\right)-r\left(X_{2}\right)
$$

Hence,

$$
X_{1}=X_{2} \Longleftrightarrow \mathcal{R}\left(X_{1}\right)=\mathcal{R}\left(X_{2}\right), \quad \mathcal{R}\left(X_{1}^{*}\right)=\mathcal{R}\left(X_{2}^{*}\right)
$$

Obviously, the matrix $(A B)^{\dagger}$ is an outer inverse of $A B$ by the definition of the MoorePenrose inverse. It is also easy to verify that $B^{\dagger}\left(A^{\dagger} A B B^{\dagger}\right)^{\dagger} A^{\dagger}$ is an outer inverse of $A B$. Thus, it follows by (44) that

$$
\begin{aligned}
r\left[(A B)^{\dagger}-B^{\dagger}\left(A^{\dagger} A B B^{\dagger}\right)^{\dagger} A^{\dagger}\right] \\
=r\left[\begin{array}{c}
(A B)^{\dagger} \\
B^{\dagger}\left(A^{\dagger} A B B^{\dagger}\right)^{\dagger} A^{\dagger}
\end{array}\right]+r\left[(A B)^{\dagger}, B^{\dagger}\left(A^{\dagger} A B B^{\dagger}\right)^{\dagger} A^{\dagger}\right] \\
\quad-r\left[(A B)^{\dagger}\right]-r\left[B^{\dagger}\left(A^{\dagger} A B B^{\dagger}\right)^{\dagger} A^{\dagger}\right] .
\end{aligned}
$$

Hence, one can derive from (44) that (39) holds if and only if

$$
\mathcal{R}\left[(A B)^{\dagger}\right]=\mathcal{R}\left[B^{\dagger}\left(A^{\dagger} A B B^{\dagger}\right)^{\dagger} A^{\dagger}\right], \quad \mathcal{R}\left\{\left[(A B)^{\dagger}\right]^{*}\right\}=\mathcal{R}\left\{\left[B^{\dagger}\left(A^{\dagger} A B B^{\dagger}\right)^{\dagger} A^{\dagger}\right]^{*}\right\} .
$$

It is also easy to verify that

$$
\begin{gathered}
r\left[\begin{array}{c}
(A B)^{\dagger} \\
B^{\dagger}\left(A^{\dagger} A B B^{\dagger}\right)^{\dagger} A^{\dagger}
\end{array}\right]=r\left[A B, A A^{*} A B\right], \\
r\left[(A B)^{\dagger}, B^{\dagger}\left(A^{\dagger} A B B^{\dagger}\right)^{\dagger} A^{\dagger}\right]=r\left[\begin{array}{c}
A B \\
A B B^{*} B
\end{array}\right], \\
r\left[(A B)^{\dagger}\right]=r\left[B^{\dagger}\left(A^{\dagger} A B B^{\dagger}\right)^{\dagger} A^{\dagger}\right]=r(A B) .
\end{gathered}
$$

Then, (43) follows. Another rank equality related to (39) is

$$
r\left[(A B)^{\dagger}-B^{\dagger}\left(A^{\dagger} A B B^{\dagger}\right)^{\dagger} A^{\dagger}\right]=r\left[(A B)^{\dagger}-\left(A^{\dagger} A B\right)^{\dagger} A^{\dagger}\right]+r\left[(A B)^{\dagger}-B^{\dagger}\left(A B B^{\dagger}\right)^{\dagger}\right]
$$

which is shown in [26]. Two rank formulas associated with (40) are given below. 
TheOREM 6. Let $A \in \mathbb{C}^{m \times n}$ and $B \in \mathbb{C}^{n \times p}$. Then,

$$
\begin{aligned}
r\left[(A B)^{\dagger}-B^{*}\left(A^{*} A B B^{*}\right)^{\dagger} A^{*}\right]= & r\left[\begin{array}{c}
(A B)^{\dagger} \\
B^{*}\left(A^{*} A B B^{*}\right)^{\dagger} A^{*}
\end{array}\right] \\
& +r\left[(A B)^{\dagger}, B^{*}\left(A^{*} A B B^{*}\right)^{\dagger} A^{*}\right] \\
& -r\left[(A B)^{\dagger}\right]-r\left[B^{*}\left(A^{*} A B B^{*}\right)^{\dagger} A^{*}\right], \\
r\left[(A B)^{\dagger}-B^{*}\left(A^{*} A B B^{*}\right)^{\dagger} A^{*}\right]= & r\left[\begin{array}{c}
A B \\
A B B^{*} B
\end{array}\right]+r\left[A B, A A^{*} A B\right]-2 r(A B) .
\end{aligned}
$$

Hence, the following statements are equivalent:

(a) (40) holds,

(b) $\mathcal{R}\left[(A B)^{\dagger}\right]=\mathcal{R}\left[B^{*}\left(A^{*} A B B^{*}\right)^{\dagger} A^{*}\right], \mathcal{R}\left\{\left[(A B)^{\dagger}\right]^{*}\right\}=\mathcal{R}\left\{\left[B^{*}\left(A^{*} A B B^{*}\right)^{\dagger} A^{*}\right]^{*}\right\}$,

(c) (14) holds.

Proof. Note that $B^{*}\left(A^{*} A B B^{*}\right)^{\dagger} A^{*}(A B) B^{*}\left(A^{*} A B B^{*}\right)^{\dagger} A^{*}=B^{*}\left(A^{*} A B B^{*}\right)^{\dagger} A^{*}$, that is, $B^{*}\left(A^{*} A B B^{*}\right)^{\dagger} A^{*}$ is an outer inverse of $A B$. Thus, (50) is derived from (44), and (51) is a simplification of (50).

Applying (13), (43), and (51) to the two products $A^{\dagger} A B$ and $A B B^{\dagger}$ gives us the following result.

TheOREM 7. Let $A \in \mathbb{C}^{m \times n}$ and $B \in \mathbb{C}^{n \times p}$, and let $T=\left(I_{n}-B B^{\dagger}\right)\left(I_{n}-A^{\dagger} A\right)$. Then,

$$
\begin{aligned}
& r\left[\left(A^{\dagger} A B\right)^{\dagger}-B^{\dagger} A^{\dagger} A+B^{\dagger} T^{\dagger} A^{\dagger} A\right]=r\left[\begin{array}{c}
A B \\
A B B^{*} B
\end{array}\right]-r(A B), \\
& r\left[\left(A B B^{\dagger}\right)^{\dagger}-B B^{\dagger} A^{\dagger}+B B^{\dagger} T^{\dagger} A^{\dagger}\right]=r\left[A B, A A^{*} A B\right]-r(A B), \\
& r\left[\left(A^{\dagger} A B\right)^{\dagger}-B^{\dagger}\left(A^{\dagger} A B B^{\dagger}\right)^{\dagger} A^{\dagger} A\right]=r\left[\begin{array}{c}
A B \\
A B B^{*} B
\end{array}\right]-r(A B), \\
& r\left[\left(A B B^{\dagger}\right)^{\dagger}-B B^{\dagger}\left(A^{\dagger} A B B^{\dagger}\right)^{\dagger} A^{\dagger}\right]=r\left[A B, A A^{*} A B\right]-r(A B), \\
& r\left[\left(A^{\dagger} A B\right)^{\dagger}-B^{*}\left(A^{\dagger} A B B^{*}\right)^{\dagger} A^{\dagger} A\right]=r\left[\begin{array}{c}
A B \\
A B B^{*} B
\end{array}\right]-r(A B), \\
& r\left[\left(A B B^{\dagger}\right)^{\dagger}-B B^{\dagger}\left(A^{*} A B B^{\dagger}\right)^{\dagger} A^{*}\right]=r\left[A B, A A^{*} A B\right]-r(A B),
\end{aligned}
$$

Hence,

(a) the following statements are equivalent:
(i) $\left(A^{\dagger} A B\right)^{\dagger}=B^{\dagger} A^{\dagger} A-B^{\dagger} T^{\dagger} A^{\dagger} A$,
(ii) $\left(A^{\dagger} A B\right)^{\dagger}=B^{\dagger}\left(A^{\dagger} A B B^{\dagger}\right)^{\dagger}$,
(iii) $\left(A^{\dagger} A B\right)^{\dagger}=B^{*}\left(A^{\dagger} A B B^{*}\right)^{\dagger}$,
(iv) $\mathcal{R}\left[\left(A B B^{*} B\right)^{*}\right]=\mathcal{R}\left[(A B)^{*}\right]$,

(b) the following statements are equivalent:

(i) $\left(A B B^{\dagger}\right)^{\dagger}=B B^{\dagger} A^{\dagger}-B B^{\dagger} T^{\dagger} A^{\dagger}$,

(ii) $\left(A B B^{\dagger}\right)^{\dagger}=\left(A^{\dagger} A B B^{\dagger}\right)^{\dagger} A^{\dagger}$,

(iii) $\left(A B B^{\dagger}\right)^{\dagger}=\left(A^{*} A B B^{\dagger}\right)^{\dagger} A^{*}$,

(iv) $\mathcal{R}\left(A A^{*} A B\right)=\mathcal{R}(A B)$. 
The combination of (4), (14), (39), (40), (41), (47) and Theorems 6 and 7 gives us the following result.

THEOREM 8. Let $A \in \mathbb{C}^{m \times n}$ and $B \in \mathbb{C}^{n \times p}$, and let $T=\left(I_{n}-B B^{\dagger}\right)\left(I_{n}-A^{\dagger} A\right)$. Then, the following statements are equivalent:

(a) $(A B)^{\dagger}=B^{\dagger}\left(A^{\dagger} A B B^{\dagger}\right)^{\dagger} A^{\dagger}$,

(b) $\left(A^{\dagger} A B B^{\dagger}\right)^{\dagger}=B^{\dagger}(A B)^{\dagger} A^{\dagger}$,

(c) $(A B)^{\dagger}=\left(A^{\dagger} A B\right)^{\dagger} A^{\dagger}=B^{\dagger}\left(A B B^{\dagger}\right)^{\dagger}$,

(d) $\left(A^{\dagger} A B\right)^{\dagger}=(A B)^{\dagger} A$ and $\left(A B B^{\dagger}\right)^{\dagger}=B(A B)^{\dagger}$,

(e) $\left(A^{\dagger} A B\right)^{\dagger}=B^{\dagger}\left(A^{\dagger} A B B^{\dagger}\right)^{\dagger}$ and $\left(A B B^{\dagger}\right)^{\dagger}=\left(A^{\dagger} A B B^{\dagger}\right)^{\dagger} A^{\dagger}$,

(f) $\left(A^{\dagger} A B\right)^{\dagger}=B^{*}\left(A^{\dagger} A B B^{*}\right)^{\dagger}$ and $\left(A B B^{\dagger}\right)^{\dagger}=\left(A^{*} A B B^{\dagger}\right)^{\dagger} A^{*}$,

(g) $(A B)^{\dagger}=B^{*}\left(A^{*} A B B^{*}\right)^{\dagger} A^{*}$,

(h) $\left(A^{*} A B B^{*}\right)^{\dagger}=\left(B^{*}\right)^{\dagger}(A B)^{\dagger}\left(A^{*}\right)^{\dagger}$,

(i) $(A B)^{\dagger}=B^{\dagger} A^{\dagger}-B^{\dagger} T^{\dagger} A^{\dagger}$,

(j) $\left(A^{\dagger} A B\right)^{\dagger}=B^{\dagger} A^{\dagger} A-B^{\dagger} T^{\dagger} A^{\dagger} A$ and $\left(A B B^{\dagger}\right)^{\dagger}=B B^{\dagger} A^{\dagger}-B B^{\dagger} T^{\dagger} A^{\dagger}$,

(k) $\mathcal{R}\left[(A B)^{\dagger}\right]=\mathcal{R}\left(B^{\dagger} T_{1}^{\dagger} A^{\dagger}\right)$ and $\mathcal{R}\left\{\left[(A B)^{\dagger}\right]^{*}\right\}=\mathcal{R}\left[\left(B^{\dagger} T_{1}^{\dagger} A^{\dagger}\right)^{*}\right]$, where $T_{1}=$ $A^{\dagger} A B B^{\dagger}$

(l) $\mathcal{R}\left[(A B)^{\dagger}\right]=\mathcal{R}\left(B^{*} T_{2}^{\dagger} A^{*}\right)$ and $\mathcal{R}\left\{\left[(A B)^{\dagger}\right]^{*}\right\}=\mathcal{R}\left[\left(B^{*} T_{2}^{\dagger} A^{*}\right)^{*}\right]$, where $T_{2}=$ $A^{*} A B B^{*}$,

(m) $\mathcal{R}\left[\left(A^{*}\right)^{\dagger} B\right]=\mathcal{R}(A B)$ and $\mathcal{R}\left(B^{\dagger} A^{*}\right)=\mathcal{R}\left[(A B)^{*}\right]$,

(n) $\mathcal{R}\left(A A^{*} A B\right)=\mathcal{R}(A B)$ and $\mathcal{R}\left[\left(A B B^{*} B\right)^{*}\right]=\mathcal{R}\left[(A B)^{*}\right]$.

The results given above can be extended to the weighted Moore-Penrose inverse of a matrix product. The weighted Moore-Penrose inverse of a matrix $A \in \mathbb{C}^{m \times n}$ with respect to a pair of Hermitian positive definite matrices $M \in \mathbb{C}^{m \times m}$ and $N \in \mathbb{C}^{n \times n}$ is defined to be the unique $n \times m$ matrix that satisfies the following four matrix equations:

(i) $A X A=A$,

(ii) $X A X=X$,

(iii) $(M A X)^{*}=M A X$,

(iv) $(N X A)^{*}=N X A$,

and this $X$ is denoted as $X=A_{M, N}^{\dagger}$. In particular, when $M=I_{m}$ and $N=I_{n}, A_{I_{m}, I_{n}}^{\dagger}$ is the standard Moore-Penrose inverse $A^{\dagger}$ of $A$. Reverse-order laws for the weighted MoorePenrose inverse of matrix products have been studied; see [17, 24, 27]. As is well known (see, e.g., [2]), the weighted Moore-Penrose inverse $A_{M, N}^{\dagger}$ of $A$ can be rewritten as

$$
A_{M, N}^{\dagger}=N^{-1 / 2}\left(M^{1 / 2} A N^{-1 / 2}\right)^{\dagger} M^{1 / 2}
$$

where $M^{1 / 2}$ and $N^{1 / 2}$ are the positive definite square roots of $M$ and $N$, respectively. By appealing to (12), one can obtain the following basic rank formula:

$$
r\left(D-C A_{M, N}^{\dagger} B\right)=r\left[\begin{array}{cc}
A^{*} M A N^{-1} A^{*} & A^{*} M B \\
C N^{-1} A^{*} & D
\end{array}\right]-r(A) ;
$$

see also [26]. Applying Theorem 8 to $\left(M^{1 / 2} A B N^{-1 / 2}\right)^{\dagger}$ in

$$
(A B)_{M, N}^{\dagger}=N^{-1 / 2}\left(M^{1 / 2} A B N^{-1 / 2}\right)^{\dagger} M^{1 / 2},
$$


and noting that

$$
A_{M, I}^{\dagger}=\left(M^{1 / 2} A\right)^{\dagger} M^{1 / 2}, \quad B_{I, N}^{\dagger}=N^{-1 / 2}\left(B N^{-1 / 2}\right)^{\dagger}
$$

yields the following result.

TheOREM 9. Let $A \in \mathbb{C}^{m \times n}$ and $B \in \mathbb{C}^{n \times p}$, and let $M \in \mathbb{C}^{m \times m}$ and $N \in \mathbb{C}^{p \times p}$ be a pair of Hermitian positive definite matrices. Then, the following statements are equivalent:

(a) $(A B)_{M, N}^{\dagger}=B_{I, N}^{\dagger}\left(A_{M, I}^{\dagger} A B B_{I, N}^{\dagger}\right)^{\dagger} A_{M, I}^{\dagger}$,

(b) $(A B)_{M, N}^{\dagger}=\left(A_{M, P}^{\dagger} A B\right)_{P, N}^{\dagger} A_{M, P}^{\dagger}=B_{P, N}^{\dagger}\left(A B B_{P, N}^{\dagger}\right)_{M, P}^{\dagger}$,

(c) $(A B)_{M, N}^{\dagger}=N^{-1} B^{*}\left(A^{*} M A B N^{-1} B^{*}\right)^{\dagger} A^{*} M$,

(d) $(A B)_{M, N}^{\dagger}=B_{I, N}^{\dagger} A_{M, I}^{\dagger}-B_{I, N}^{\dagger}\left[\left(I_{n}-B B_{I, N}^{\dagger}\right)\left(I_{n}-A_{M, I}^{\dagger} A\right)\right]^{\dagger} A_{M, I}^{\dagger}$,

(e) $\mathcal{R}\left(A A^{*} M A B\right)=\mathcal{R}(A B)$ and $\mathcal{R}\left[\left(A B N^{-1} B^{*} B\right)^{*}\right]=\mathcal{R}\left[(A B)^{*}\right]$.

REMARK 10. Reverse-order laws can be established from any reasonable operations for the Moore-Penrose inverse of matrix products. For example, write

$$
A B=A A^{\dagger} A B B^{\dagger} B=A A^{*}\left(A^{\dagger}\right)^{*}\left(B^{\dagger}\right)^{*} B^{*} B=A A^{*}\left[\left(A^{\dagger}\right)^{*}\left(B^{\dagger}\right)^{*}\right] B^{*} B:=P N Q
$$

The reverse-order law $(P N Q)^{\dagger}=Q^{\dagger} N^{\dagger} P^{\dagger}$ is equivalent to

$$
(A B)^{\dagger}=\left(B^{*} B\right)^{\dagger}\left[\left(B^{\dagger} A^{\dagger}\right)^{\dagger}\right]^{*}\left(A A^{*}\right)^{\dagger} .
$$

The law $(A B)^{\dagger}=B^{*}\left(A^{*} A B B^{*}\right)^{\dagger} A^{*}$ is obtained from writing

$$
A B=\left(A^{*}\right)^{\dagger}\left(A^{*} A B B^{*}\right)\left(B^{*}\right)^{\dagger}:=P N Q
$$

and $(P N Q)^{\dagger}=Q^{\dagger} N^{\dagger} P^{\dagger}$. On the other hand, mixed-type reverse-order laws can be introduced by comparing different decompositions of a block matrix and their generalized inverses. The introduction of (4) is such an example. In addition, one can consider some variations of (4). For instance, replacing the Moore-Penrose inverses in (4) with $\{1\}$-inverses yields the following reverse-order law for $(A B)^{-}$:

$$
(A B)^{-}=B^{-} A^{-}-B^{-} F_{A}\left(E_{B} F_{A}\right)^{-} E_{B} A^{-},
$$

where $F_{A}=I_{n}-A^{-} A$ and $E_{B}=I_{n}-B B^{-}$. One can also establish some rank equalities associated with this reverse-order law and then derive necessary and sufficient conditions for this law to hold.

For a triple matrix product $A B C$, the Moore-Penrose inverse $(A B C)^{\dagger}$ can be written as either $(A B C)^{\dagger}=C^{\dagger} B^{\dagger} A^{\dagger}$ or $(A B C)^{\dagger}=C^{\dagger} B^{\dagger} A^{\dagger}+X$. The law $(A B C)^{\dagger}=C^{\dagger} B^{\dagger} A^{\dagger}$ was studied in $[10,19]$. Necessary and sufficient conditions for this law to hold are quite strict and complicated. Two reasonable extensions of (4) to $(A B C)^{\dagger}$ are

$$
\begin{aligned}
& (A B C)^{\dagger}=(B C)^{\dagger} B(A B)^{\dagger}-(B C)^{\dagger}\left[P_{B C}^{\perp} B P_{(A B)^{*}}^{\perp}\right]^{\dagger}(A B)^{\dagger}, \\
& (A B C)^{\dagger}=C^{\dagger}\left[I_{p}-\left(P_{C}^{\perp} P_{B^{*}}^{\perp}\right)^{\dagger}\right] B^{\dagger}\left[I_{n}-\left(P_{B}^{\perp} P_{A^{*}}^{\perp}\right)^{\dagger}\right] A^{\dagger}
\end{aligned}
$$


Some reasonable extensions of (39) and (40) to $(A B C)^{\dagger}$ are

$$
\begin{gathered}
(A B C)^{\dagger}=C^{\dagger}\left(A^{\dagger} A B C C^{\dagger}\right)^{\dagger} A^{\dagger}, \\
(A B C)^{\dagger}=C^{*}\left(A^{*} A B C C^{*}\right)^{\dagger} A^{*}, \\
(A B C)^{\dagger}=(B C)^{\dagger}\left[(A B)^{\dagger} A B C(B C)^{\dagger}\right]^{\dagger}(A B)^{\dagger} .
\end{gathered}
$$

Various rank formulas associated with these reverse-order laws can be established. For more details, see [18].

ACKNOWLEDGMENTS. The author would like to express his sincere thanks to Professors G. P. H. Styan, S. Drury, X.-W. Chang, and Y. Takane at McGill University for their supports during the preparation of this paper. Sincere thanks also are due to the referees for the helpful comments and suggestions.

\section{REFERENCES}

[1] J. K. Baksalary, Algebraic characterizations and statistical implications of the commutativity of orthogonal projectors, Proceedings of the Second International Tampere Conference in Statistics (T. Pukkila and S. Puntanen, eds.), University of Tampere, Tampere, 1987, pp. 113-142.

[2] A. Ben-Israel and T. N. E. Greville, Generalized Inverses. Theory and Applications, 2nd ed., CMS Books in Mathematics, vol. 15, Springer-Verlag, New York, 2003.

[3] D. Buckholtz, Hilbert space idempotents and involutions, Proc. Amer. Math. Soc. 128 (2000), no. 5, 1415-1418.

[4] S. L. Campbell and C. D. Meyer, Jr., Generalized Inverses of Linear Transformations, Dover Publications, New York, 1991, corrected reprint of the 1979 original.

[5] S. Cheng and Y. Tian, Moore-Penrose inverses of products and differences of orthogonal projectors, Acta Sci. Math. (Szeged) 69 (2003), no. 3-4, 533-542.

[6] R. E. Cline, Note on the generalized inverse of the product of matrices, SIAM Rev. 6 (1964), 57-58.

[7] R. E. Cline and T. N. E. Greville, An extension of the generalized inverse of a matrix, SIAM J. Appl. Math. 19 (1970), 682-688.

[8] A. M. Galperin and Z. Waksman, On pseudo-inverses of operator products, Linear Algebra Appl. 33 (1980), 123-131.

[9] T. N. E. Greville, Note on the generalized inverse of a matrix product, SIAM Rev. 8 (1966), 518-521.

[10] R. E. Hartwig, The reverse order law revisited, Linear Algebra Appl. 76 (1986), 241-246.

[11] S. Izumino, The product of operators with closed range and an extension of the reverse order law, Tôhoku Math. J. (2) 34 (1982), no. 1, 43-52.

[12] G. Marsaglia and G. P. H. Styan, Equalities and inequalities for ranks of matrices, Linear and Multilinear Algebra 2 (1974/75), 269-292.

[13] T. Oikhberg, Products of orthogonal projections, Proc. Amer. Math. Soc. 127 (1999), no. 12, 3659-3669.

[14] R. Penrose, A generalized inverse for matrices, Math. Proc. Cambridge Philos. Soc. 51 (1955), 406-413.

[15] V. Rakočević, On the norm of idempotent operators in a Hilbert space, Amer. Math. Monthly 107 (2000), no. 8, 748-750.

[16] C. R. Rao and S. K. Mitra, Generalized Inverse of Matrices and Its Applications, John Wiley \& Sons, New York, 1971.

[17] W. Sun and Y. Wei, Inverse order rule for weighted generalized inverse, SIAM J. Matrix Anal. Appl. 19 (1998), no. 3, 772-775. 
[18] Y. Tian, Some mixed-type reverse order laws for the Moore-Penrose inverse of a triple product, to appear in Rocky Mountain J. Math.

[19] _ Reverse order laws for the generalized inverses of multiple matrix products, Linear Algebra Appl. 211 (1994), 85-100.

[20] _ How to characterize equalities for the Moore-Penrose inverse of a matrix, Kyungpook Math. J. 41 (2001), no. 1, 1-15.

[21] _ Problem 29-10: Equivalence of three reverse-order laws, Image (The Bulletin of the International Linear Algebra Society) 29 (2002), 35, Solutions by J. K. Baksalary and O. M. Baksalary, O. M. Baksalary and K. Chylińska, S. Cheng, Y. Tian, and H. J. Werner, Image (The Bulletin of the International Linear Algebra Society) 30 (2003), 29-31.

[22] _ Rank equalities related to outer inverses of matrices and applications, Linear and Multilinear Algebra 49 (2002), no. 4, 269-288.

[23] _ Upper and lower bounds for ranks of matrix expressions using generalized inverses, Linear Algebra Appl. 355 (2002), 187-214.

[24] _ Reverse order laws for the weighted Moore-Penrose inverse of a triple matrix product with applications, Int. Math. J. 3 (2003), no. 1, 107-117.

[25] _ Rank equalities for block matrices and their Moore-Penrose inverses, Houston J. Math. 30 (2004), 483-510.

[26] __ Using rank formulas to characterize equalities for Moore-Penrose inverses of matrix products, Appl. Math. Comput. 147 (2004), no. 2, 581-600.

[27] Y. Tian and S. Cheng, Some identities for Moore-Penrose inverses of matrix products, Linear and Multilinear Algebra, in press.

[28] S. Zlobec, An explicit form of the Moore-Penrose inverse of an arbitrary complex matrix, SIAM Rev. 12 (1970), 132-134.

Yongge Tian: Department of Mathematical and Statistical Sciences, University of Alberta, Edmonton, AB, Canada T6G 2G1

E-mail address: ytian@stat. ualberta.ca 


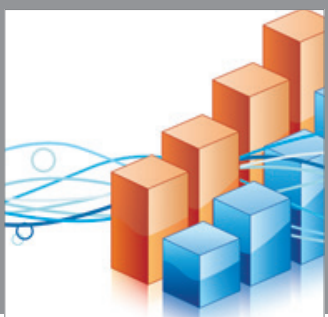

Advances in

Operations Research

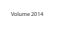

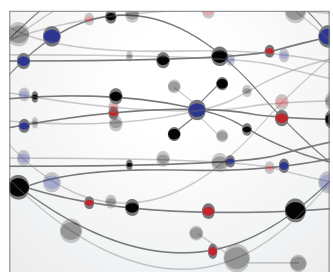

\section{The Scientific} World Journal
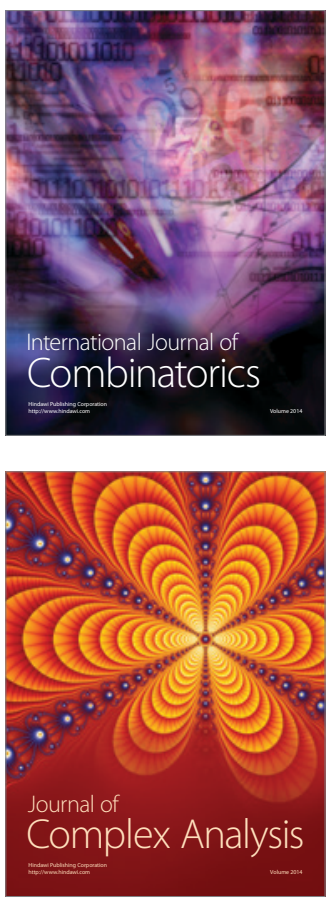

International Journal of

Mathematics and

Mathematical

Sciences
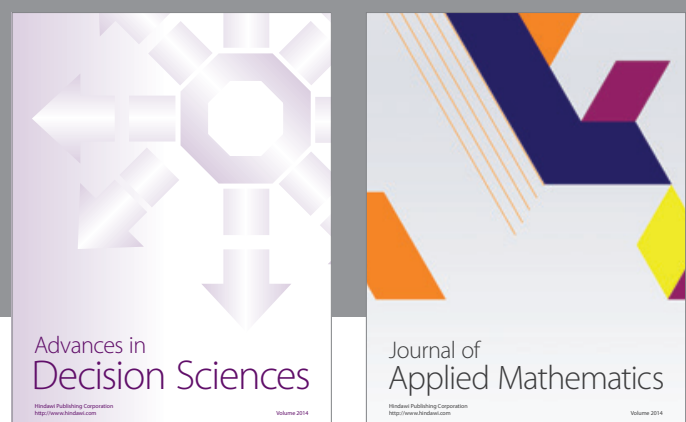

Journal of

Applied Mathematics
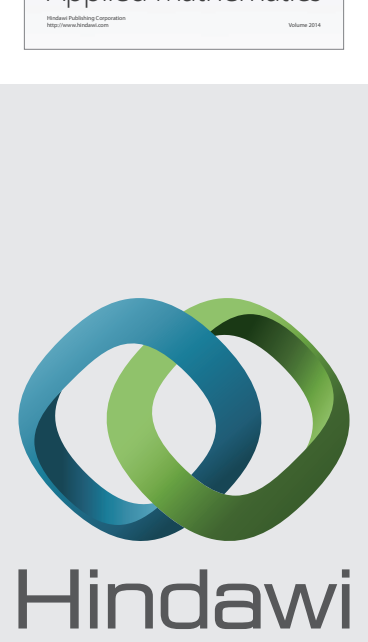

Submit your manuscripts at http://www.hindawi.com
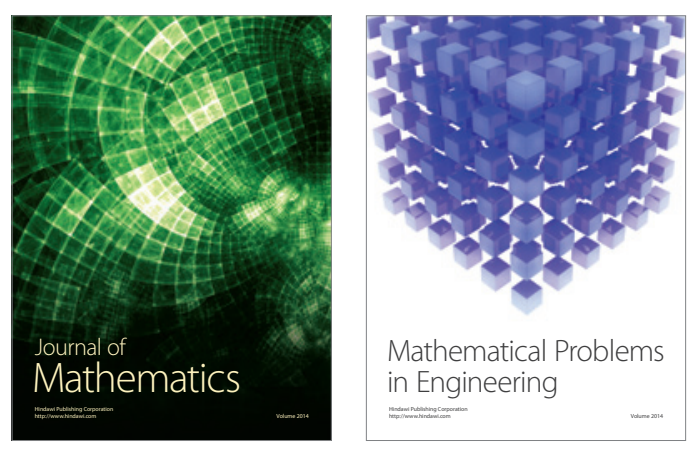

Mathematical Problems in Engineering
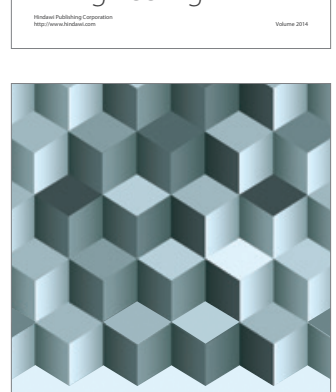

Journal of

Function Spaces
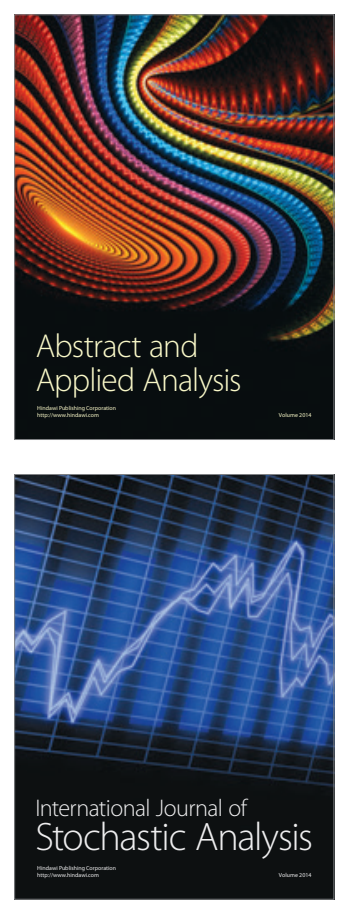

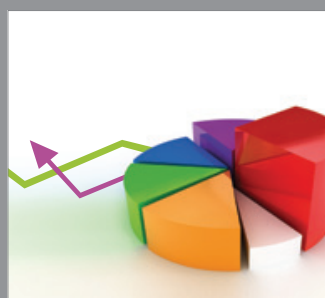

ournal of

Probability and Statistics

Promensencen
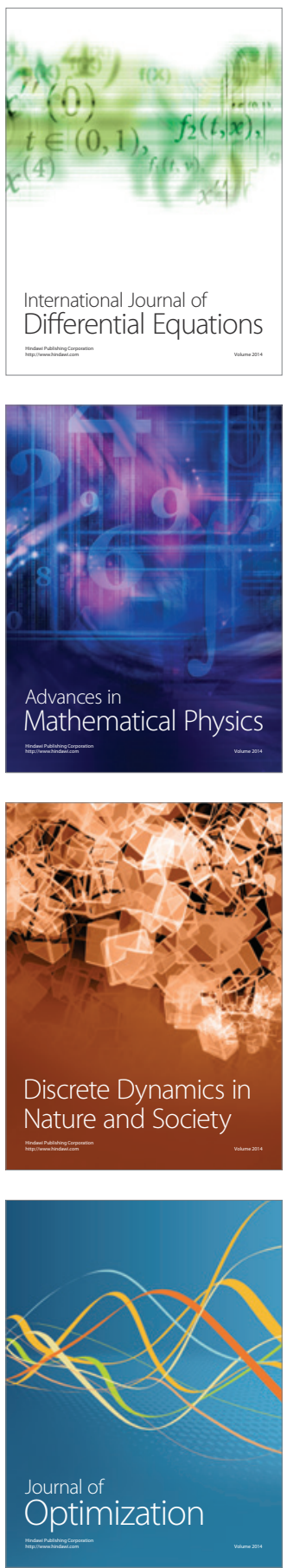